\title{
Influence of Operating Conditions and Material Properties on the Mass Transport Losses of Polymer Electrolyte Water Electrolysis
}

\author{
Michel Suermann, ${ }^{\text {a,* }}$ Kazuhiro Takanohashi, ${ }^{\text {a,c }}$ Adrien Lamibrac, ${ }^{\text {a }}$ Thomas J. Schmidt,,${ }^{\text {a,b,**}}$ \\ and Felix N. Büchi ${ }^{\mathbf{a}, \mathbf{z}}$
}

${ }^{a}$ Electrochemistry Laboratory, Paul Scherrer Institut, CH-5232 Villigen PSI, Switzerland

${ }^{b}$ Laboratory of Physical Chemistry, ETH Zürich, CH-8093 Zürich, Switzerland

\begin{abstract}
Three different porous transport layer (PTL) structures, based on titanium sintered powders, were characterized using X-ray tomographic microscopy to determine key geometric properties such as porosity, pore and particle size distributions as well as effective transport properties. The mass transport through the PTL contributes to the voltage losses in the polymer electrolyte water electrolysis cell. Therefore, influence of the PTL structure on the mass transport overpotential is investigated as function of current densities $\left(\leq 4 \mathrm{~A} \cdot \mathrm{cm}^{-2}\right)$, operating pressures $(1-100 \mathrm{bar})$ and temperatures $\left(40-60^{\circ} \mathrm{C}\right)$, respectively. A decrease of transport losses was observed with increasing pressure and temperature for all investigated PTLs. At around 100 bar balanced pressure, the transport losses for all PTLs converge to about $40 \mathrm{mV}$ per applied A $\cdot \mathrm{cm}^{-2}$, suggesting that other parts of the cell such as the catalyst layer or their interface contribute to these remaining losses. The performance loss, induced by the different PTL structures, shows a stronger correlation with geometric parameters such as pore and particle size distributions than transport properties like effective diffusivity and permeability. The finest materials with $\mathrm{d}_{50}$ pore and particle diameters of $40-48$ and $68 \mu \mathrm{m}$, respectively, are performing better than the coarsest material with diameters roughly twice the sizes.

(C) The Author(s) 2017. Published by ECS. This is an open access article distributed under the terms of the Creative Commons Attribution 4.0 License (CC BY, http://creativecommons.org/licenses/by/4.0/), which permits unrestricted reuse of the work in any medium, provided the original work is properly cited. [DOI: 10.1149/2.13517109jes] All rights reserved.

(cc) BY
\end{abstract}

Manuscript submitted May 19, 2017; revised manuscript received July 6, 2017. Published July 18, 2017.

Polymer electrolyte water electrolysis (PEWE) is a promising technology to convert (surplus) electric energy into chemical energy in form of hydrogen to avoid curtailment of the fluctuating renewable electricity sources ${ }^{1}$ and thus reducing the need of fossil fuels. ${ }^{2}$ Depending on the use of hydrogen, the gas has to be provided at pressure levels up to 1000 bar, i.e. for the use in mobility applications. ${ }^{3}$ Commercial electrolyzers are operated at pressures in the order of $30 \mathrm{bar}$ (hydrogen and optionally oxygen) ${ }^{4}$ to reduce downstream compression and drying efforts. ${ }^{5}$

For energy applications, high efficiency of the electrolysis conversion process is a key property. As in any electrochemical cell, also with PEWE, losses/overpotentials incur when a current is passed. Ohmic and kinetic overpotentials, related to the movement of protons in the polymer electrolyte and the finite rates of the electrochemical reactions are reasonably well understood for PEWE. ${ }^{6-8}$ In most cases, at least at current densities above about $1 \mathrm{~A} \cdot \mathrm{cm}^{-2}$, losses in addition to ohmic and kinetic sources are observed and related to mass transport resistances in the porous structures of the catalyst and porous transport layers (PTLs), though their nature and origin in PEWE are not well understood. ${ }^{6}$ Nevertheless, as long as a sufficient water supply is guaranteed, no transport limitations occur in the form of a turning point in the current/voltage characteristics (i/E-curves), as demonstrated by Lewinski et al. ${ }^{9}$ up to $19 \mathrm{~A} \cdot \mathrm{cm}^{-2}$.

In polymer electrolyte fuel cells (PEFC), a technology similar to PEWE, the effect of mass transport in the micro-porous structures of the electrodes and PTLs on the performance of the cells is reasonably well studied. In particular the transport resistances in the PTLs (termed gas diffusion layers, GDLs, in PEFC) is well understood, e.g. due to flooding of the cathodic GDL, and may be a limiting factor in PEFC at high current densities. ${ }^{10,11}$

In PEWE, PTLs are also used between the flow-field structures and the catalyst layers (CLs). As in fuel cells, PTLs provide simultaneous transport of charge and heat in the solid, and gas and water in the pore space. In case differential pressure is applied, the PTL has furthermore to provide mechanical support for the membrane and electrodes. Due to the high potential at the anode in electrolysis, the corrosive nature of deionized water and the acidic environment of the polymer elec-

\footnotetext{
*Electrochemical Society Student Member.

**Electrochemical Society Member.

Permanent Address: University of Yamanashi, 6-43 Miyamae, JP-400-0021 Kofu, Japan.

${ }^{\mathrm{z} E-m a i l: ~ f e l i x . b u e c h i @ p s i . c h ~}$
}

trolyte, commonly (anode) PTLs based on titanium powders, fibers or expanded sheets are used. However, only in few studies the influence of different titanium based materials on PEWE performance has been investigated as summarized below.

PTLs prepared from titanium sintered spherical powders, with particle diameters in the $50-75 \mu \mathrm{m}$ range and porosities in the $28-40 \%$ range were investigated by Grigoriev et al. ${ }^{12}$ They used a mathematical approach based on packing density and particle sizes to calculate mean pore diameter and distribution. The samples were characterized electrochemically up to $2 \mathrm{~A} \cdot \mathrm{cm}^{-2}$ at ambient pressure and $90^{\circ} \mathrm{C}$. The authors claim that the optimal mean pore diameter should be between $12-13 \mu \mathrm{m}$ and the optimal porosity in the $30-50 \%$ range for sintered spherical powder materials. However, materials with higher porosities were not studied. The optimum mean pore diameter was considered a compromise between capillary effects, which become relevant at pore sizes below about $10 \mu \mathrm{m}$, and additional parasitic ohmic losses for larger particle diameters.

Ito et al. ${ }^{13,14}$ investigated titanium felt based PTLs up to $1 \mathrm{~A} \cdot \mathrm{cm}^{-2}$ at ambient pressure and $80^{\circ} \mathrm{C}$. Different fiber diameters $(20,40$ and $80 \mu \mathrm{m}$ ) were partly decorated with titanium powder to achieve porosities of 46-75\% and average pore diameters in the 12-106 $\mu \mathrm{m}$ range. The average pore diameter was assesed by determination of the breakthrough point. From the electrochemical performance point of view, an average pore diameter of greater than $10 \mu \mathrm{m}$, but as close as possible to this size, was considered as best. With respect to porosity, no significant influence was observed for values above $50 \%$. However, no samples with smaller average pore diameter and lower porosity were investigated. Nevertheless, the authors highlighted that if the average pore diameter exceeds $90 \mu \mathrm{m}$, which is the case only for the $80 \mu \mathrm{m}$ fiber material, a slightly increased ohmic resistance was measured.

A complementary study by Hwang et al. ${ }^{15}$ in the field of unitized reversible fuel cells (URFCs) revealed that additional PTFE loading/coating of titanium felt based materials increased the fuel cell, but did not affect the electrolysis performance, respectively.

The above summarized literature results are based on average values for pore and particle size distributions only and no attempt was made to characterize the structure in more detail. In the PEFC community, X-ray tomographic microscopy (XTM) is common practice to characterize the structure and/or water distribution and transport in carbon fiber based GDLs. ${ }^{16,17}$ Zielke et al. ${ }^{18}$ have applied the same technique to determine geometric and transport properties of the dry PTL structures made from different titanium sintered powders and fibers. Independent of the samples, the authors found a 
relation between through-plane electric conductivity and permeability and an exponential relation between porosity and thermal conductivity. Unfortunately, the corresponding electrochemical characterization is missing.

By changing the ionomer loading in the anodic $\mathrm{CL}$ and the cathodic pressure, Bernt and Gasteiger ${ }^{19}$ showed that the proton conductivity in and gas removal from the porous CL and PTL structures contribute to the transport overpotential.

In addition to the electrochemical characterization and dry structure properties of the PTL materials, also their gas and water transport characteristics are of interest, especially at the anode, where gas and water move in counter-flow. ${ }^{7}$ This two-phase flow was visualized by visible light, neutron and X-ray imaging with focus of the PTL/flowfield interface and the flow regime in the channels. ${ }^{20-23}$ Based on operando in-plane neutron radiography data, Seweryn et al. ${ }^{24}$ reported a gas/water ratio in the PTL independent of current density, verified up to $2.5 \mathrm{~A} \cdot \mathrm{cm}^{-2}$. However, in none of these studies flow properties were correlated with electrochemical performance.

The present study, therefore aims at closing part of this gap for a range of titanium sinter powder materials. The geometric and transport properties of three different materials are characterized with ex situ XTM and the data are correlated with electrochemical performance based on galvanostatic i/E-curves combined with high frequency resistance (HFR) measurements. The correlation of PTL structure and electrochemical performance may vary with operating conditions. Therefore, the electrochemical performances are analyzed in the temperature range of $40-60^{\circ} \mathrm{C}$ for current densities up to $4 \mathrm{~A} \cdot \mathrm{cm}^{-2}$ and pressures up to 100 bar. The aim of the work is to get a better understanding of the PTL influence on the transport overpotential providing input for manufacturers and additional experimental and theoretical work.

\section{Experimental}

Materials.-Three different PTLs made from titanium sintered powder materials (SIKA T5, T10 and T20, GKN Sinter Metal Filters, DE) with 1 and $2 \mathrm{~mm}$ nominal thickness are investigated. The actual thicknesses deviate between $-2 \%$ to $+25 \%$ from the nominal thickness. The hydrophilic PTL's raw material complies with titanium grade 2 . As the materials have initially been developed for filtering, the numbering, therefore, codes the maximum particle diameter (in $\mu \mathrm{m}$ ) passing the material, i.e. can be interpreted as the bottleneck size in the through-plane pore structure.

Electrolysis cell.-Commercial catalyst coated membranes (CCM, Nafion 117CS, Greenerity E400, Greenerity, DE) were used for electrochemical evaluation. A differential cell (no gradients along the channel) with an active area of $4 \mathrm{~cm}^{2}$ was used. Flow-fields on both sides had identical structures with 5 straight parallel channels (20 mm long, $1 \mathrm{~mm}$ height, $2 \mathrm{~mm}$ width) and $2 \mathrm{~mm}$ ribs. With a set of springs between the cathodic flow-field and the distributor plate, a defined contact pressure in the active area (in this work $2.5 \mathrm{MPa}$ ) is set independently of the clamping pressure.

Test bench.-An electrolysis test bench with two separate gas/water loops is used for testing. At the anode water is recirculated by a diaphragm pump with a defined water flow of about 30 $\mathrm{mL} \cdot \mathrm{min}^{-1} \cdot \mathrm{cm}^{-2}$. At the cathode natural convection takes place. The reference and operating cell temperature is measured by a thermocouple inside a drill hole in the cell housing close to the active area and controls heat exchangers at the gas/water separators. The test bench and electrolysis cell are described in detail in Ref. 25.

Measurement setup.-All electrochemical measurements were performed using a potentiostat (VSP-300, Bio-Logic SAS, FR). A current density of $2 \mathrm{~A} \cdot \mathrm{cm}^{-2}$ was chosen for equilibration of at least ten minutes before performing $\mathrm{i} / \mathrm{E}$-curves; $2 \mathrm{~A} \cdot \mathrm{cm}^{-2}$ are later referred to as the reference current density. At a constant current density, little or no change in the cell voltage over several hours is observed.
The i/E-curves were recorded between 0.001 and $4 \mathrm{~A} \cdot \mathrm{cm}^{-2}$ with relatively short holding times of $10 \mathrm{~s}$ plus $1 \mathrm{~s}$ HFR measurement. The chosen holding time is a compromise between (thermal) equilibrium conditions and safety due to gas crossover at high pressures and low current densities, as validated earlier. ${ }^{26}$ The HFR is measured at $25 \mathrm{kHz}$.

Generally, one measurement series was carried out per material, except for T10-1mm, which was tested in 3 cells. The results are listed as the mean values and labeled as T10-1mm (underlined font). For each material/operating parameter combination at least two, but commonly three $\mathrm{i} / \mathrm{E}$-curves were measured and the average and standard deviation is reported in the graphs.

X-ray tomographic microscopy imaging.-XTM of the dry PTLs was performed using a CT-scanner (phoenix nanotom $m$, General Electric, DE) with sample specific measurement parameters given in Supplementary Table 1 . The sample size resulted from the determination of the representative elementary volume (REV), as described later, and needed to be chosen larger for the coarsest material. The chosen region of interest (RoI) is smaller than the sample size considering only the bulk material and thus excluding surface/edge effects. The voxel edge length is a result of the sample size and its distance to the detector. A copper filter was used to suppress beam hardening effects by reducing the soft part of the X-ray spectrum. The total measurement time $\left(t_{\mathrm{tot}}\right)$ is a function of the number of projections per scan $(N)$, exposure time $\left(t_{\exp }\right)$ and the number of average and skipped projections $(n+s)$ and is about two hours per scan.

Image processing.-Reconstruction of the XTM scans results in gray scale 3D images. These gray scale images are segmented into two phases, i.e. solid and void, using the software ImageJ/Fiji. The largely different X-ray attenuation coefficients of titanium and air result in a clear contrast between solid and void. For optimization of image processing, a mean filter is applied before segmenting the two phases using the Otsu threshold method. ${ }^{27}$

Region of interest.-The cutting edges of the titanium samples are partly melted and deformed from the wire cutting process. The surfaces have slightly different properties as a result of the production process. Therefore, the cutting edges and the surfaces are excluded from the RoI for determining the geometric and transport properties. Thus, the calculated structural properties are representative for the bulk phase.

Geometric properties.-All geometric and transport properties were determined for the bulk material. ImageJ/Fiji was used to determine the geometric properties of porosity and the solid (particle) and void (pore) size distributions (SSD and VSD). Both, SSD and VSD were determined using the 2D local thickness method ${ }^{28}$ as a quantitative parameter. Local thickness describes the particle and pore size dimensions by fitting the largest possible disc in the structure, for any pixel of the respective phase.

Transport properties.-For determining transport properties, the segmented XTM images were further processed using the software package GeoDict (Math2Market, DE). Boundary conditions were set as described below. For the permeability a linear flow solution method with a periodic repetition plus a 20 voxels thick inflow region, tested from 5 to 80 voxels, was set. Permeability (unit $\mathrm{m}^{2}$ ) is given a material property by considering separately the fluid viscosity in the Darcy's law. Concerning the effective diffusivity and tortuosity, a bulk diffusion $(\mathrm{Kn} \ll 1)$ solution method solving the flow direction in any direction and periodic boundaries were chosen. The Knudsen number $(\mathrm{Kn})$ is defined as ratio of the mean free path to the representative physical length. Electric and thermal conductivities were calculated for $20^{\circ} \mathrm{C}$ using Dirichlet boundary conditions. For titanium, the electric and thermal conductivity were set to $2.38 \cdot 10^{6} \mathrm{~S} / \mathrm{m}$ and 
$21.9 \mathrm{~W} \cdot(\mathrm{m} \cdot \mathrm{K})^{-1}$, respectively. For liquid water the thermal conductivity is $0.6 \mathrm{~W} \cdot(\mathrm{m} \cdot \mathrm{K})^{-1}$.

Representative elementary volume.-The REV, i.e. the minimum volume representing the same characteristics as the bulk material, ${ }^{29,30}$ is determined for the different structures and porosity. For statistics, cubes with varying cube edge lengths from 200 up to $1500 \mu \mathrm{m}$ were implemented into the RoI, similar to Ref. 31, without overlapping as summarized in Supplementary Table 2. The target criterion was set to a relative error, i.e. the ratio of the standard deviation to the average of the porosity, of less or equal to $5 \%$ to ensure a representative material analysis with minimized computing time. For instance, in Supplementary Figure 1A the mean, minimum and maximum porosity of T10 sample together with the corresponding relative error is given as a function of the cube edge length. When considering porosity and the target criterion of a relative error of less or equal to $5 \%$, the REV cube edge length corresponds to about $400 \mu \mathrm{m}$. With respect to the geometric and transport properties calculated with Geodict (permeability, effective diffusivity, tortuosity, electric and thermal conductivity), only one cube with varying edge length is used to reduce computing time. For statistical purposes, the three space directions $(x, y, z)$ are compared as a function of the cube edge length, as shown in Supplementary Figure 1B for the T10 samples' effective diffusivity. All curves (x,y,z and mean) converge at about $400 \mu \mathrm{m}$ cube edge length, similar to the REV obtained before for porosity. This agreement is valid for all investigated parameters. Thus, the REV cube edge length for both the T5 and T10 sample was chosen to be $0.4 \mathrm{~mm}$ and for the T20 sample $1 \mathrm{~mm}$. In the Results section, all geometric and transport properties presented are based on calculations for these REV dimensions.

\section{Results}

The Results section is separated into two main topics: i) characterization of the dry PTL structures, analyzing the geometric and transport properties of the three different sinter materials and ii) characterization of the corresponding electrochemical performance of cells with the three structures as function of current density, pressure and temperature. Where possible, correlations between the two topics are made and discussed with respect to literature results.

Characterization of the dry PTL structure.-Geometric properties.-The segmented images of the different particulate sinter materials are illustrated in 3D in Figure 1. Based on the segmentation process, black and white correspond to void and solid, respectively.

Based on the XTM data, the porosity of the three materials is similar. The values for T5, T10 and T20 are $30 \pm 2 \%, 35 \pm 2 \%$, and $33 \pm 2 \%$, respectively, as summarized in Table I. When comparing results of the 2D local thickness analysis for all three space directions $(\mathrm{x}, \mathrm{y}, \mathrm{z})$, no significant differences for any sample were observed. The structures of the sinter materials are therefore considered isotropic.

\begin{tabular}{|c|c|c|c|c|c|c|}
\hline Property & \multicolumn{2}{|c|}{$\mathrm{T} 5$} & \multicolumn{2}{|c|}{$\mathrm{T} 10$} & \multicolumn{2}{|c|}{$\mathrm{T} 20$} \\
\hline $\begin{array}{l}\text { Tortuosity } \tau[-] \\
\text { Porosity } \varepsilon[\%]\end{array}$ & \multicolumn{2}{|c|}{$\begin{array}{c}1.9 \pm 0.1 \\
30 \pm 2\end{array}$} & \multicolumn{2}{|c|}{$\begin{array}{c}1.6 \pm 0.1 \\
35 \pm 2\end{array}$} & \multicolumn{2}{|c|}{$\begin{array}{c}1.8 \pm 0.1 \\
33 \pm 2\end{array}$} \\
\hline Size distribution $[\mu \mathrm{m}]$ & void & solid & void & solid & void & solid \\
\hline $\mathrm{d}_{10}$ & 16 & 34 & 20 & 34 & 32 & 48 \\
\hline $\mathrm{d}_{50}$ & 40 & 68 & 48 & 68 & 80 & 128 \\
\hline $\mathrm{d}_{90}$ & 68 & 110 & 82 & 132 & 144 & 232 \\
\hline Modal & 18 & 46 & 22 & 44 & 16 & 56 \\
\hline Median & 28 & 52 & 32 & 52 & 48 & 80 \\
\hline Mean & 30 & 56 & 38 & 52 & 57 & 96 \\
\hline
\end{tabular}

Hence, if not mentioned otherwise, the average of all directions for any geometric and transport property is given.

The local thickness distributions are compared in Figure 2A for the void and in Figure $2 \mathrm{C}$ for the solid phase. For the void, only the open pores are considered, though the ratio of the closed pores is negligibly small $(<0.5 \%$ closed porosity). The results show that T5 and T10 have quite sharp and almost identical SSD with a maximum at about $50 \mu \mathrm{m}$. The materials seem to be made from the same powders. For the VSD, a shift to larger pores is observed for T10 with maxima for T5 at $18 \mu \mathrm{m}$ and T10 at $22 \mu \mathrm{m}$. In contrast T20 is sintered from a different powder with significantly larger particles with a median particle diameter of $80 \mu \mathrm{m}$ compared to T5 and T10 with $52 \mu \mathrm{m}$ and has therefore also a VSP with a significant fraction of larger pores (Table I).

When VSD and SSD are plotted as cumulative distributions, the differences can be seen more clearly as illustrated in Figures $3 \mathrm{~B}$ and $3 D$. From the cumulative plots, the $d_{10}, d_{50}$ and $d_{90}$ values of pore and particle size distribution are determined and summarized in Table I. The $d_{n}$ values describe the size at which $n \%$ of the pores/particles are smaller than the $d_{n}$ value.

Normalized on the same cumulative VSD, generally the pores of T5 are slightly smaller compared to T10. In contrast, the corresponding T20 pore diameter is almost doubled. As already seen from the distributed size distributions, particles of T5 and T10 are equal up to about $80 \%$ of the cumulative SSD, e.g. $\mathrm{d}_{50}$ is $68 \mu \mathrm{m}$ for both materials. In contrast, the particle size of T20 is shifted to about twice the size with $\mathrm{d}_{50}=128 \mu \mathrm{m}$. The cumulative VSDs show similar characteristics with T20 having about twice the sizes for $\mathrm{d}_{10}$ and $\mathrm{d}_{50}$ of $\mathrm{T} 5$ and T10. All materials consist of a large number of relatively small pores $(<40$ $\mu \mathrm{m})$, but their contribution to the cumulative volume is low. It can be summarized that for T5 and T10 identical powder material are used and only the sintering conditions are varied to generate the different VSDs. In contrast, for T20, bigger particle size powders are used to
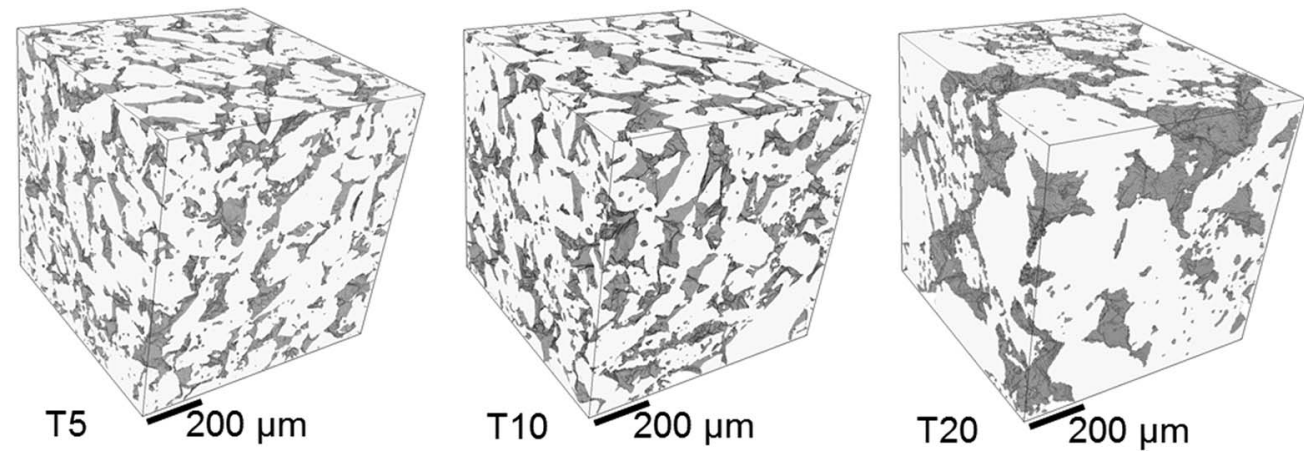

Figure 1. 3D images of T5, T10 and T20 after XTM image processing with white (solid) and black (void) phases. 

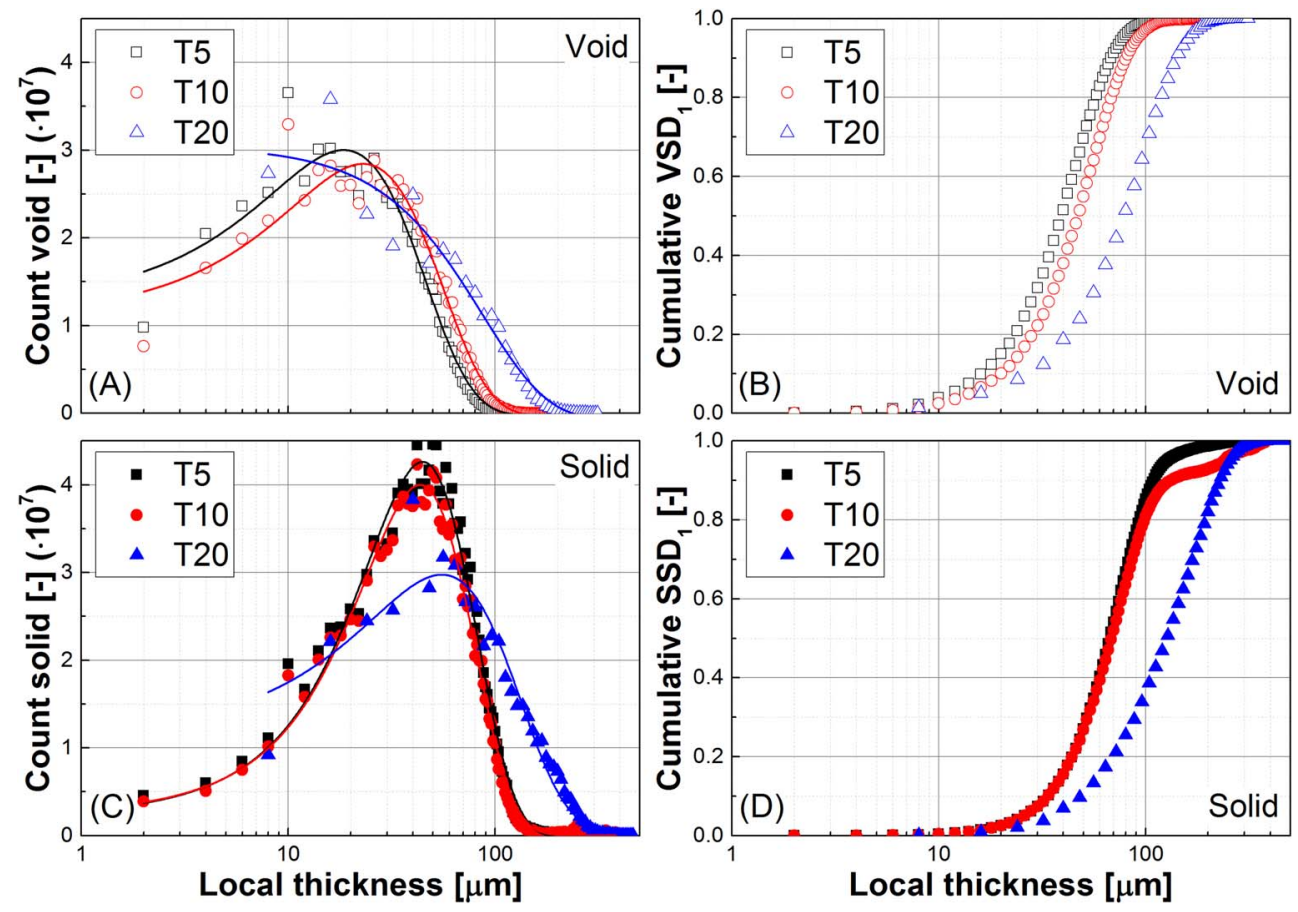

Figure 2. 2D local thickness analysis for T5, T10 and T20; (A) and (B) for the pore space, (C) and (D) the particle phase; (A) and (C) show histograms for the diameter; for illustration the distributions are fitted with the Giddings peak function; (B) and (D) show the corresponding cumulative size distributions.

obtain bigger pores by maintaining a porosity value in-between $\mathrm{T} 5$ and $\mathrm{T} 10$.

Besides VSD and SSD also the tortuosity is an important structural property, which can have significant influence on the transport properties of a porous material. For the tortuosity $\tau$, i.e. the ratio of the effective path length to the shortest distance between two points in the porous material, in the void phase, no trend with powder or particle sizes is observed. The tortuosities of the materials are $1.8 \pm$ 0.2 (Table I), without significant difference between the materials.

In conclusion, the three materials have significantly different properties for the pore sizes and peak of the VSD, e.g. the pore $\mathrm{d}_{50}$ values increase with the formal classification. Other structural properties such as the porosity and tortuosity are within a small range for the three materials and do not show a significant trend.
Transport properties.-Transport through porous structures can be described with effective transport properties. The effective properties of interest are the permeability and the effective diffusivity. Permeability is a descriptor for the pressure driven flow in porous materials and is therefore an important property for both gas and water transport in the PTL. Permeability increases from T5 to T20 from 1.4 to $7.7 \cdot 10^{-12} \mathrm{~m}^{2}$ (Table II). As for the pressure driven flow, pore size is an important parameter, the increase of the permeability is consistent with the increasing pore sizes (Table I) of the investigated PTLs.

In a porous medium, the effective diffusion coefficient of species is different from the diffusion coefficient in the open space, due to a reduced available cross section for diffusion. The effective diffusion coefficient $D_{\text {eff }}$ of a species in a porous material can also be used as descriptor for the porous structure and being determined according to
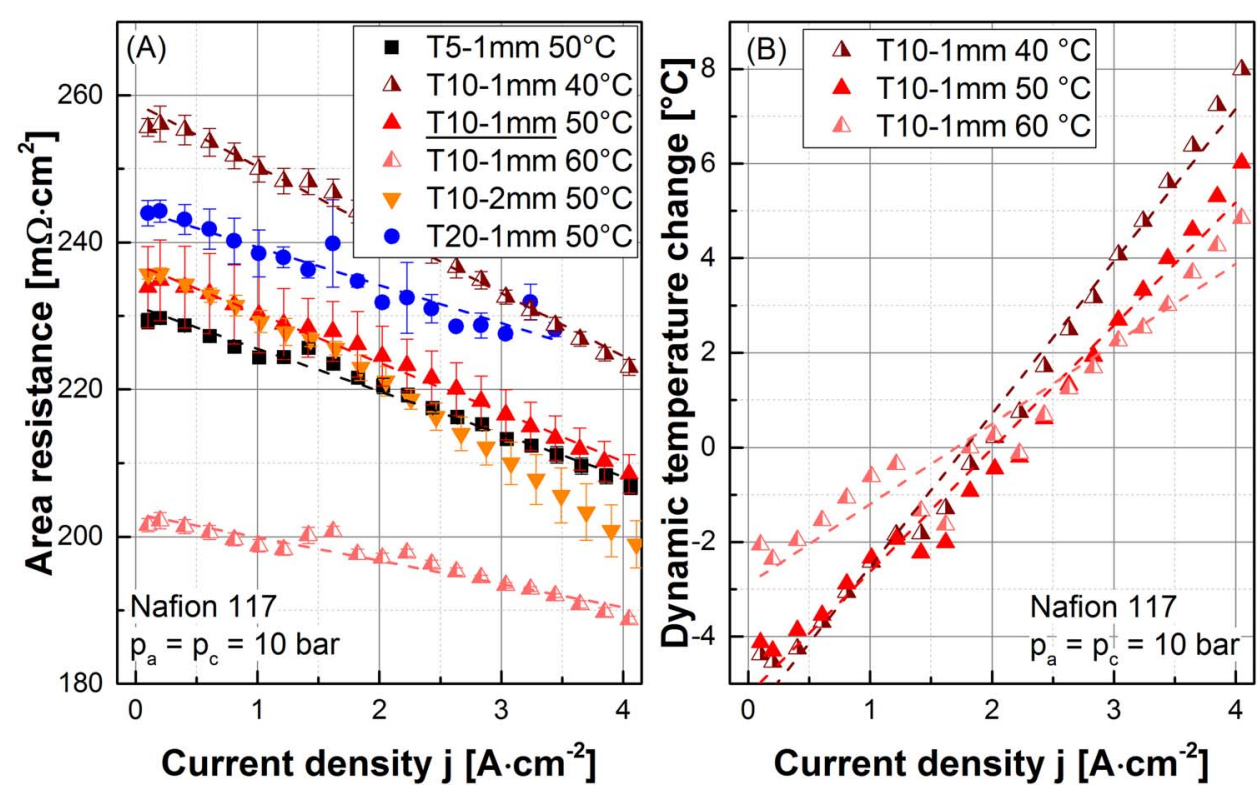

Figure 3. (A) Area resistance vs. current density for different PTLs using Nafion $117 \mathrm{mem}$ branes at $50^{\circ} \mathrm{C}$ and 10 bar balanced pressure. For one out of the three T10-1mm samples, data for 40 and $60^{\circ} \mathrm{C}$ is added too. The HFR was measured at $25 \mathrm{kHz}$. Measurement points below $0.1 \mathrm{~A} \cdot \mathrm{cm}^{-2}$ were removed for clarity. (B) Calculated dynamic temperature change vs. current density for T10-1mm, based on an Arrhenius behavior as explained in Ref. 26. 
Table II. Transport properties for the bulk material of T5, T10 and T20. The given values correspond to the average and the standard deviation of the three space directions due to the isotropic nature of the sinter metals.

\begin{tabular}{|c|c|c|c|c|}
\hline Property & unit & T5 & $\mathrm{T} 10$ & $\mathrm{~T} 20$ \\
\hline $\begin{array}{l}\text { Effective } \\
\text { diffusivity }\end{array}$ & - & $0.09 \pm 0.01$ & $0.14 \pm 0.01$ & $0.09 \pm 0.01$ \\
\hline Permeability & $\cdot 10^{-12} \mathrm{~m}^{2}$ & $1.4 \pm 0.0$ & $3.4 \pm 0.1$ & $7.7 \pm 0.2$ \\
\hline $\begin{array}{l}\text { Electric } \\
\text { conductivity }\end{array}$ & $\cdot 10^{6} \mathrm{~S} / \mathrm{m}$ & $1.2 \pm 0.0$ & $0.9 \pm 0.1$ & $1.1 \pm 0.1$ \\
\hline $\begin{array}{l}\text { Thermal } \\
\text { conductivity }\end{array}$ & $\mathrm{W} \cdot(\mathrm{m} \cdot \mathrm{K})^{-1}$ & $11.6 \pm 0.3$ & $9.6 \pm 0.4$ & $11.0 \pm 0.7$ \\
\hline
\end{tabular}

Equation 1:

$$
D_{\text {bulk }}=D_{\text {bulk }}^{0} \cdot D_{\text {eff }} \quad \text { with } \quad D_{\text {eff }}=\frac{\varepsilon}{\tau^{2}}
$$

where $D^{0}{ }_{\text {bulk }}$ is the diffusion coefficient in the empty space, $\varepsilon$ is the porosity, $\tau$ is the tortuosity. ${ }^{32}$ The effective diffusivities for all three materials are in the order of 0.1 and summarized in Table II.

For the solid phase, the electric and thermal conductivities are of interest; values are given in Table II. The specific electric resistance of the bulk phase is approximately $1 \mathrm{~m} \Omega \cdot \mathrm{cm}$ and thus its influence on performance negligibly small for the investigated thicknesses of 1 and $2 \mathrm{~mm}$. More important are the thermal conductivities which are for all materials in the order of $10 \mathrm{~W} /(\mathrm{m} \cdot \mathrm{K})$, as this property can strongly influence the temperature gradients in the membrane electrode assembly (MEA). With each Watt of thermal power per square centimeter, a temperature gradient of about $1 \mathrm{~K}$ appears for the $1 \mathrm{~mm}$ thick PTLs with the given thermal conductivities.

Electrochemical characterization of the porous transport layer.-Cells with three different PTLs (T5, T10 and T20), always with the same type at anode and cathode, have been electrochemically characterized as a function of the current density, operating pressure and for T10 also for temperature. The transport overpotential is extracted from $\mathrm{i} / \mathrm{E}$-curve data.

Overpotential analysis.-The cell voltage is composed of the sum of the thermodynamic cell voltage $E_{\text {cell }}^{\circ}(\mathrm{p}, \mathrm{T})$ and three main overpotentials, i.e. kinetic $\eta_{\text {kin }}$, ohmic $\eta_{\mathrm{iR}}$ and mass transport $\eta_{\mathrm{mtx}}$, according to Equation 2:

$$
E_{\text {cell }}=E_{\text {cell }}^{0}(p, T)+\eta_{\mathrm{kin}}+\eta_{\mathrm{iR}}+\eta_{\mathrm{mtx}}
$$

The thermodynamic cell voltage is derived from the Nernst Equation 3:

$$
E_{\text {cell }}^{0}(p, T)=E^{0}(T)+\frac{R \cdot T}{2 \cdot F} \ln \left(\frac{\left.a\left(\mathrm{H}_{2}\right) \cdot \sqrt{a\left(\mathrm{O}_{2}\right.}\right)}{a\left(\mathrm{H}_{2} \mathrm{O}\right)}\right)
$$

where $\alpha$ is the activity of the species and $E^{0}(T)$ is the temperature dependent equilibrium potential. ${ }^{33}$ Ideal gas behavior is assumed for the activity of the gases. ${ }^{34-36}$ The activity of liquid water is set to unity.

The ohmic overpotential is calculated from the HFR measurements. To distinguish between the kinetic and mass transport overpotentials, an analysis method based on the Tafel model is applied. At low current densities $\left(0.01-0.1 \mathrm{~A} \cdot \mathrm{cm}^{-2}\right)$, where no mass transport overpotential is expected, a Tafel line (Equation 4) is fitted in the linear region and extrapolated toward higher current densities:

$$
\eta_{\text {kin }}=b \cdot \log \left(\frac{j}{j_{0}}\right)
$$

with $b$ being the Tafel slope and $j_{0}$ the apparent exchange current density. Consequently, the kinetic overpotential is equal to the difference between the extrapolated Tafel line and the thermodynamic cell voltage and the mass transport overpotential corresponds to the difference between the iR-free cell voltage and the extrapolated Tafel line. Thus, the transport overpotential includes anodic and cathodic contributions, whereas the two-phase counter-flow at the anode is most likely the crucial side. All $\mathrm{i} / \mathrm{E}$-curves were analyzed in the same manner by the above-described method.

Ohmic overpotential.-The HFR (measured at $25 \mathrm{kHz}$ ) is plotted in Figure $3 \mathrm{~A}$ against current density for cells with different PTLs. For all curves, a slight decrease of the area resistance with increasing current density is observed as a result of slight MEA temperature increase with increasing current density due to limited heat conductivity of the porous PTL structures. For the $2 \mathrm{~mm}$ thick T10 sample, the effect is even more pronounced due to the higher thermal resistance.

From the HFR measurements at the reference current density of $2 \mathrm{~A} \cdot \mathrm{cm}^{-2}$ at different operating temperatures, an Arrhenius behavior (apparent activation energy $E_{\mathrm{a}}$ for proton conduction of about 10 $\mathrm{kJ} / \mathrm{mol}$ ) can be deduced, which is in good agreement with previous measurements in the $30-70^{\circ} \mathrm{C}$ range. ${ }^{26}$ The method is explained in detail in Ref. 26. Thus, the actual CCM temperature can be determined from the HFR measurements which could not be measured with a thermocouple close to the active area within the cell housing. Exemplarily shown for T10-1mm in Figure 3B, at current densities smaller than the reference current density, a cooling effect of up to $-4^{\circ} \mathrm{C}$ appears. While at current densities higher than $2 \mathrm{~A} \cdot \mathrm{cm}^{-2}$ a heating effect up to $+8^{\circ} \mathrm{C}$ is observed. Both the cooling and the heating effect are more pronounced at lower cell temperatures, because of higher overpotentials at a given current density.

Since all experiments were performed with the same type of CCM, based on Nafion 117, differences in the HFR outside the statistical error must be attributed to varying PTL properties. A slight shift to higher area resistances of about $10 \mathrm{~m} \Omega \cdot \mathrm{cm}^{2}$ between T20 and the rest (T5 and T10) is observed. This shift cannot be explained by the bulk electric conductivity of the PTLs (Table II). Consequently, other characteristics have to come into focus, e.g. the SSD, where T20 has clearly bigger particles with a $\mathrm{d}_{50}$ of $128 \mu \mathrm{m}$ compared to $\mathrm{T} 10$ and T5 with $68 \mu \mathrm{m}$ each (Table I). Because the differences in HFR cannot be explained by the PTL bulk properties, it is hypothesized that the different SSD results in different interfacial contact resistances (ICRs) between PTLs and CLs. ICRs may be quantified using ex situ methods, ${ }^{37}$ as demonstrated for PEWE PTLs by Lettenmeier et al. ${ }^{38}$ who reported a reduction in the ICR of about $20 \mathrm{~m} \Omega \cdot \mathrm{cm}^{2}$ when adding a microporous layer (MPL) to the PTL (also T10).

Transport overpotential.-The iR-free $\mathrm{i} / \mathrm{E}$-curves at $50^{\circ} \mathrm{C}$ and 10 bar balanced pressure for cells with each of the three PTL types are shown in Figure 4A. Figure 4B shows the corresponding Tafel plots. The Tafel slopes coincide at $70 \pm 2 \mathrm{mV} / \mathrm{dec}$. Consequently, the differences in the electrochemical performance of the cells with different PTL types are observed in the mass transport overpotential. T20 clearly generates higher mass transport overpotentials than T5 and T10 at a given current density. Furthermore, there seems to be a trend that T10 performs slightly better than T5, however, the difference lies within the standard deviation. The same holds true for the influence of the thickness, investigated for the T10 samples with 1 and $2 \mathrm{~mm}$ thickness.

Influence of pressure.-The PTL induced difference in the mass transport overpotential is investigated as a function of pressure at $50^{\circ} \mathrm{C}$. Results for 1 and $3 \mathrm{~A} \cdot \mathrm{cm}^{-2}$ are shown in Figure 5 , for the 1-100 bar balanced pressure range. Linear fits (in a semi-logarithmic plot) are added for the two current densities as function of pressure and the corresponding slopes, i.e. reduction of transport losses per decade of pressure, are summarized in Table III. Based on the data presented in Figures 4 and 5 the following findings can be formulated:

- The mass transport overpotential appears clearly starting in the range of $0.4-0.6 \mathrm{~A} \cdot \mathrm{cm}^{-2}$ and increases with current density for all investigated PTL materials and operating parameter combinations.

- The transport losses decrease with balanced pressure independent of the PTL, e.g. for T10 for both thicknesses (1 and $2 \mathrm{~mm}$ ) 15 

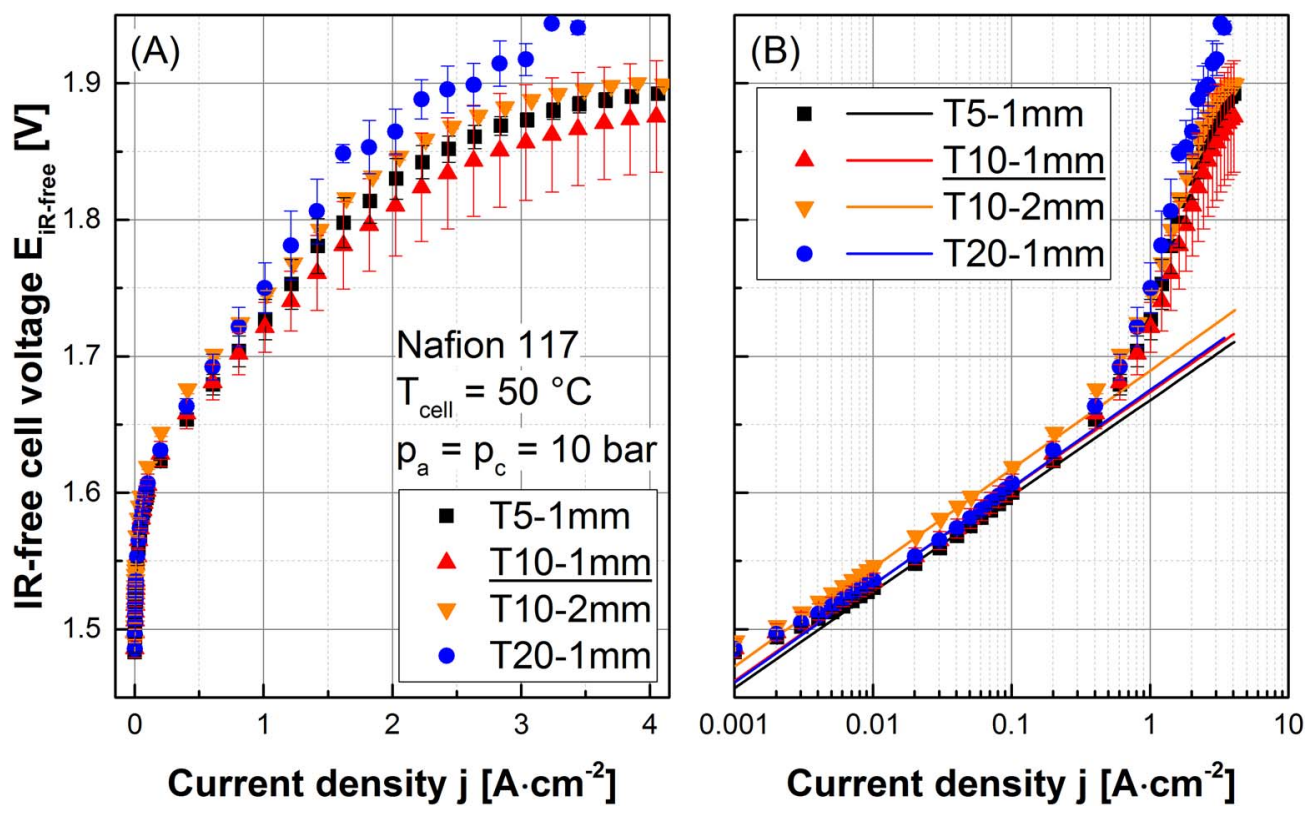

Figure 4. (A) iR-free i/E-curves for the investigated PTLs at $50^{\circ} \mathrm{C}$ and 10 bar balanced pressure; (B) corresponding Tafel plot including the fitted and extrapolated Tafel lines.

to $20 \mathrm{mV}$ per decade of balanced pressure in the investigated current range. For T5 and especially for T20 the absolute savings are even higher, especially at the higher current density.

- The differences of the mass transport overpotential between the investigated sinter metals decrease with pressure. Interestingly, the transport overpotential converge at balanced pressure in the order of 100 bar with remaining losses of about $130 \mathrm{mV}$ at $3 \mathrm{~A} \cdot \mathrm{cm}^{-2}$ and 40 $\mathrm{mV}$ at $1 \mathrm{~A} \cdot \mathrm{cm}^{-2}$.

The decreasing transport losses with pressure may be explained due to gas volume, increased water/gas ratio or solubility. The (extrapolated) fits of the transport overpotential in Figure 5 converge at about $40 \mathrm{mV}$ per applied $\mathrm{A} \cdot \mathrm{cm}^{-2}$ at a pressure in the order of $100 \mathrm{bar}$. Apparently at the elevated pressure the structural differences become

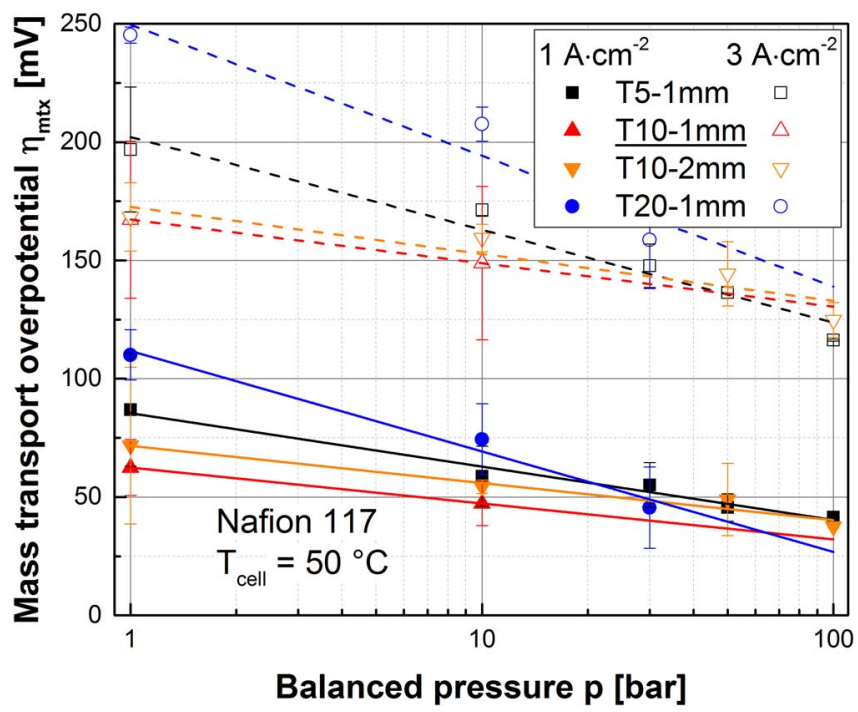

Figure 5. Comparison of the mass transport overpotential for the different types of PTL at $50^{\circ} \mathrm{C}$ as a function of balanced pressure between 1 and 100 bar at $1 \mathrm{~A} \cdot \mathrm{cm}^{-2}$ (filled symbols) and $3 \mathrm{~A} \cdot \mathrm{cm}^{-2}$ (open symbols). The data is fitted and (partly) extrapolated to 100 bar. The slope of the linear fits is given in Table III in $\mathrm{mV} / \mathrm{dec}(\mathrm{p})$. less relevant for the transport process behavior. Eventually, the remaining transport losses are not attributable to the PTL bulk structures, but rather to the interfaces with the CLs or to the porous CLs at all (both anode and cathode).

Summarizing, T20 performs electrochemically worse than T5 and $\mathrm{T} 10$, especially at lower pressure. Concerning T5 and T10 no clear difference in the overpotential analysis is observed. Combining those electrochemical findings with the results of the structural analysis, the similar electrochemical behavior of T5 and T10 is in line with an almost identical SSD and only a small shift to bigger pores for T10 compared to T5, e.g. with a void $\mathrm{d}_{50}$ of $40 \mu \mathrm{m}$ (T5) compared to 48 $\mu \mathrm{m}$ (T10). The differences in the transport properties, however, with T10 having a higher effective diffusivity $(+60 \%)$ and permeability $(+240 \%)$ are not reflected in the electrochemical performance and thus must either not be the limiting parameters and/or are superimposed by other effects. In particular for T20, with a larger VSD, e.g. void $\mathrm{d}_{50}$ of $80 \mu \mathrm{m}$, and a higher permeability $(+550 \%$ vs. T5), but with a poorer electrochemical performance, at first glance, it seems counter-intuitive. It points to the fact that the interface to the CL is of importance for the transport overpotential or that for the two phase flow (water in counter flow to the gas at the anode) permeability is not a decisive descriptor.

Influence of temperature.-For cells with T10, the influence of cell temperature is investigated between 40 and $60^{\circ} \mathrm{C}$ at a constant pressure of 10 bar (balanced). In general, as shown in Figure 6, the mass transport overpotential decreases with temperature. These results are well in line with previous data where T10-2mm was studied in the temperature range of $30-70^{\circ} \mathrm{C} .^{26}$ The flattening of the transport losses at current densities higher than $2 \mathrm{~A} \cdot \mathrm{cm}^{-2}$, seen in Figure 6, is attributed to

Table III. Slope of the linear fits presented in Figure 5 in $\mathrm{mV} / \operatorname{dec}(\mathbf{p})$.

\begin{tabular}{lcc} 
Sample & $\begin{array}{c}\text { Slope at } 1 \mathrm{~A} \cdot \mathrm{cm}^{-2} \\
{[\mathrm{mV} / \mathrm{dec}(\mathrm{p})]}\end{array}$ & $\begin{array}{c}\text { Slope at } 3 \mathrm{~A} \cdot \mathrm{cm}^{-2} \\
{[\mathrm{mV} / \mathrm{dec}(\mathrm{p})]}\end{array}$ \\
\hline T5-1mm & -23 & -39 \\
T10-1mm & -15 & -18 \\
T10-2mm & -16 & -20 \\
T20-1mm & -42 & -55
\end{tabular}




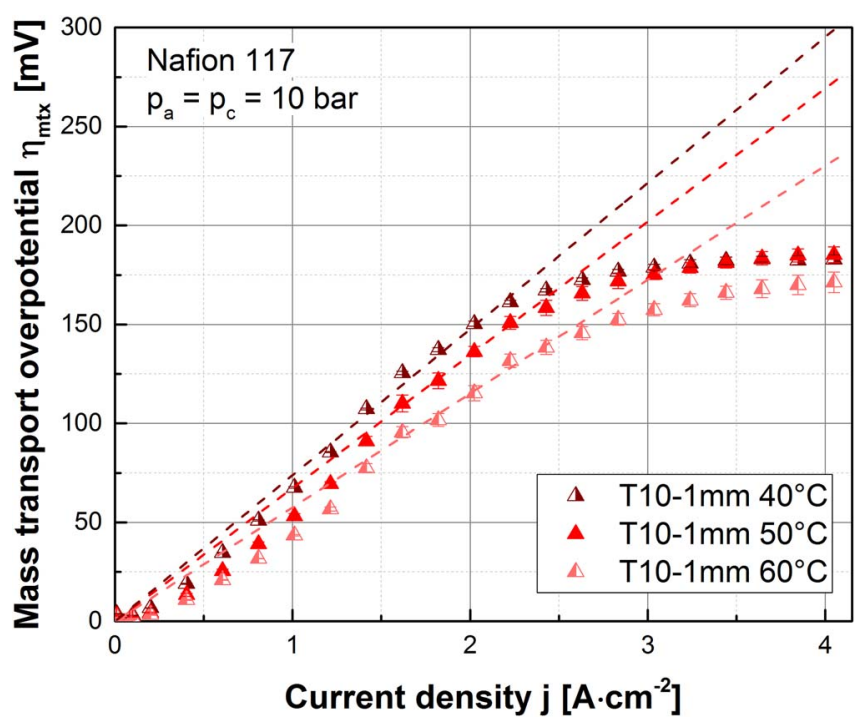

Figure 6. Influence of the temperature in the $40-60^{\circ} \mathrm{C}$ range on the mass transport overpotential for T10-1mm. Linear fits are fitted between 1.7 and 2.3 $\mathrm{A} \cdot \mathrm{cm}^{-2}$ by being forced to go through the origin and then extrapolated toward lower and higher current densities. The corresponding slopes are 74, 67 and $58 \mathrm{mV}$ per $\mathrm{A} \cdot \mathrm{cm}^{-2}$ for 40,50 and $60^{\circ} \mathrm{C}$, respectively.

an additional temperature effect due to the increasing overall overpotential with current density as described above for the HFR (Figure 3). In the current density range below $2 \mathrm{~A} \mathrm{~cm}^{-2}$, the transport overpotential has an approximately linear behavior with current density even though it is biased by the cooling effect. To disregard the thermal effect, the transport losses in Figure 6 were approximated with a linear fit (between 1.7 and $2.3 \mathrm{~A} \cdot \mathrm{cm}^{-2}$ and forced to go through the origin). The fitting current density range is chosen due to relatively small temperature deviations from the desired cell temperature (Figure $3 \mathrm{~B}$ ). The slope of the linear approximation decreases within the investigated $40-60^{\circ} \mathrm{C}$ range from 74 to $58 \mathrm{mV}$ per $\mathrm{A} \cdot \mathrm{cm}^{-2}$, indicating that for each applied $\mathrm{A} \cdot \mathrm{cm}^{-2}$, the transport losses decrease by $8 \pm$ $1 \mathrm{mV}$ per 10 degrees of increase in cell temperature.

The mechanism of the influence of the temperature on the transport losses is not clear as the reasons may lay in changes of either gas or liquid transport in the porous structure or the modification in the ICR. It can only be speculated that if the gas phase transport is limiting, the changing water partial pressure plays a key role. If the liquid phase transport is critical, then the decreasing viscosity or surface tension might influence the gas/liquid transport behavior.

A better understanding of the contribution of the PTL to the mass transport overpotential and the one of the PTL/CL interface or the CL itself, needs further investigation. However, while for today's (commercial) PEWE operating conditions in the $1-2 \mathrm{~A} \cdot \mathrm{cm}^{-2}$ current density range at temperatures of $50-80^{\circ} \mathrm{C}$ transport losses in the order of only $20-100 \mathrm{mV}$ appear, for higher current density operation in the future developments, higher mass transport overpotentials will be observed. This will require increased attention and further research to optimize PEWE efficiency in energy applications.

\section{Conclusions}

In this work, XTM was used to determine geometric and transport properties of three different PTLs made from sintered titanium powders with isotropic structure and formally different bottleneck pore sizes (5, 10 and $20 \mu \mathrm{m}$, labeled as T5, T10 and T20) and the corresponding PEWE electrochemical performances were characterized using i/E-curves and HFR measurements. For all cells with different PTLs on the one hand common general behavior of the transport losses with respect to operating conditions is observed, pointing to a common mechanism independent of the exact nature of the porous material. On the other hand, differences between the transport losses for different PTL structures are observed.

Generally, the transport overpotential shows a pseudo-linear behavior with current density in the order of $70 \pm 30 \mathrm{mV}$ per $\mathrm{A} \cdot \mathrm{cm}^{-2}$ (at ambient pressure). For each applied $\mathrm{A} \cdot \mathrm{cm}^{-2}$, this value drops by $8 \pm 1 \mathrm{mV}$ per 10 degrees of increase in cell temperature (in the 40 $60^{\circ} \mathrm{C}$ range). Water vapor pressure or the slight decrease in viscosity and/or surface tension of the liquid water may be responsible for the temperature dependence. With respect to the operating pressure, at constant current, the transport overpotential decreases significantly of about tens of millivolts per decade of pressure at relevant current densities in the $1-3 \mathrm{~A} \cdot \mathrm{cm}^{-2}$ range. Pressure dependent changes of the gas/liquid ratio in the porous structure may be responsible for this. Interestingly, the transport losses seem to converge at balanced pressure in the order of 100 bar with remaining losses of about $40 \mathrm{mV}$ per applied $\mathrm{A} \cdot \mathrm{cm}^{-2}$ which needs further investigations to dedicate it to either the structural parameters of the PTL or to other parts of the cell like the CL or their interfaces.

For the different PTL materials, it was found that the two materials with lower pore sizes are based on the same powder material $\left(\mathrm{d}_{50}\right.$ $=68 \mu \mathrm{m})$ while the T20 material is made from coarser powder $\left(\mathrm{d}_{50}\right.$ $=128 \mu \mathrm{m})$. For the pore sizes with $\mathrm{d}_{50}$ values of 40,48 and 80 $\mu \mathrm{m}$ the difference is also about a factor of 2 from the finest to the coarsest material. With respect to the transport properties of the porous materials, effective diffusivity in the void phase, and transport in the solid (heat and charge) are within $\pm 25 \%$ for all materials and therefore only limited influence on cell performance is expected. Only for the permeability an increase from finest to the coarsest material of a factor of 5 is observed, reflecting the different pore sizes.

The following correlations between structure/transport property and cell performance were identified: i) the higher HFR for the coarsest material (T20) must arise from interfacial properties with the CL due to larger particles sizes, rather than bulk conductivity; ii) the higher transport overpotential of T20 has no straight-forward explanation, as larger pore sizes and higher permeability of the PTL would rather point in the other direction. It points to the fact that also here the interface to the CL plays an important, as yet poorly understood role.

Hence, the origin of the mass transport overpotential and the critical geometric and/or transport characteristics of the PTL need further investigation, especially at the interface toward the CL. In this context, imaging techniques might help to get a better insight into the complex gas/water flow regimes.

\section{Acknowledgments}

Funding by the Swiss Federal Office of Energy (SFOE, grant no. 500904-1), Belenos Clean Power Holding Ltd. and the Energy System Integration (ESI) platform at PSI, and technical support by Martin Ammann and Thomas Gloor (both PSI), and scientific support with the XTM by Jens Eller (PSI) are gratefully acknowledged. TJS thanks the Commission for Technology and Innovation Switzerland and the Swiss Competence Center for Energy Research Heat \& Electricity Storage.

\section{References}

1. C. J. Barnhart, M. Dale, A. R. Brandt, and S. M. Benson, Energy \& Environmental Science, 6, 2804 (2013).

2. A. Ursúa, L. M. Gandía, and P. Sanchis, Proceedings of the IEEE, 100, 410 (2012).

3. S. Metz, Fuel Cells Bulletin, 2014, 12 (2014).

4. M. Felgenhauer and T. Hamacher, International Journal of Hydrogen Energy, 40 , 2084 (2015)

5. B. Bensmann, R. Hanke-Rauschenbach, G. Müller-Syring, M. Henel, and K. Sundmacher, Applied Energy, 167, 107 (2016)

6. M. Carmo, D. L. Fritz, J. Mergel, and D. Stolten, International Journal of Hydrogen Energy, 38, 4901 (2013)

7. U. Babic, M. Suermann, F. N. Büchi, L. Gubler, and T. J. Schmidt, Journal of The Electrochemical Society, 164, F387 (2017).

8. E. Fabbri, A. Habereder, K. Waltar, R. Kötz, and T. J. Schmidt, Catalysis Science and Technology, 4, 3800 (2014) 
9. K. A. Lewinski, D. F. van der Vliet, and S. M. Luopa, ECS Transactions, 69, 893 (2015).

10. A. Z. Weber and J. Newman, Chem. Rev., 104, 4679 (2004).

11. N. Nonoyama, S. Okazaki, A. Z. Weber, Y. Ikogi, and T. Yoshida, Journal of The Electrochemical Society, 158, B416 (2011).

12. S. A. Grigoriev, P. Millet, S. A. Volobuev, and V. N. Fateev, International Journal of Hydrogen Energy, 34, 4968 (2009).

13. H. Ito, T. Maeda, A. Nakano, C. M. Hwang, M. Ishida, A. Kato, and T. Yoshida, International Journal of Hydrogen Energy, 37, 7418 (2012).

14. H. Ito, T. Maeda, A. Nakano, A. Kato, and T. Yoshida, Electrochimica Acta, 100, 242 (2013).

15. C. M. Hwang, M. Ishida, H. Ito, T. Maeda, A. Nakano, A. Kato, and T. Yoshida, Journal of Power Sources, 202, 108 (2012).

16. S.-G. Kim and S.-J. Lee, Journal of Power Sources, 230, 101 (2013).

17. M. H. Shojaeefard, G. R. Molaeimanesh, M. Nazemian, and M. R. Moqaddari, International Journal of Hydrogen Energy, 41, 20276 (2016).

18. L. Zielke, A. Fallisch, N. Paust, R. Zengerle, and S. Thiele, RSC Advances, 4, 58888 (2014).

19. M. Bernt and H. A. Gasteiger, Journal of The Electrochemical Society, 163, F3179 (2016).

20. M. A. Hoeh, T. Arlt, N. Kardjilov, I. Manke, J. Barnhart, D. L. Fritz, J. Ehlert, W. Lüke, and W. Lehnert, ECS Transactions, 69, 1135 (2015).

21. M. A. Hoeh, T. Arlt, I. Manke, J. Banhart, D. L. Fritz, W. Maier, and W. Lehnert, Electrochemistry Communications, 55, 55 (2015).

22. F. Arbabi, A. Kalantarian, R. Abouatallah, R. Wang, J. S. Wallace, and A. Bazylak, Journal of Power Sources, 258, 142 (2014).
23. I. Dedigama, P. Angeli, K. Ayers, J. B. Robinson, P. R. Shearing, D. Tsaoulidis, and D. J. L. Brett, International Journal of Hydrogen Energy, 39, 4468 (2014).

24. J. Seweryn, J. Biesdorf, T. J. Schmidt, and P. Boillat, Journal of The Electrochemical Society, 163, F3009 (2016).

25. M. Suermann, A. Pătru, T. J. Schmidt, and F. N. Büchi, International Journal of Hydrogen Energy, 42, 12076 (2017).

26. M. Suermann, T. J. Schmidt, and F. N. Büchi, Electrochimica Acta, 211, 989 (2016).

27. N. Otsu, IEEE Transactions Systems Man, and Cybernetics, SMC-9 (1979).

28. T. Hildebrand and P. Rüegsegger, Journal of Microscopy, 185, 67 (1997).

29. R. Hill, Journal of the Mechanics and Physics of Solids, 11, 357 (1963).

30. T. Kanit, S. Forest, I. Galliet, V. Mounoury, and D. Jeulin, International Journal of Solids and Structures, 40, 3647 (2003).

31. E. A. Wargo, A. C. Hanna, A. Çeçen, S. R. Kalidindi, and E. C. Kumbur, Journal of Power Sources, 197, 168 (2012)

32. N. Epstein, Chemical Engineering Science, 44, 777 (1989).

33. I. Barin and G. Platzki, Thermochemical Data of Pure Substances, p. 795, VCH Verlagsgesellschaft mbH, Weinheim (1995).

34. H. Ito, T. Maeda, A. Nakano, and H. Takenaka, International Journal of Hydrogen Energy, 36, 10527 (2011).

35. R. Wiebe and V. L. Gaddy, Journal of the American Chemical Society, 56, 76 (1934).

36. D. Tromans, Hydrometallurgy, 48, 327 (1998).

37. H. Wang, M. A. Sweikart, and J. A. Turner, Journal of Power Sources, 115, 243 (2003).

38. P. Lettenmeier, S. Kolb, F. Burggraf, A. S. Gago, and K. A. Friedrich, Journal of Power Sources, 311, 153 (2016). 